\title{
Juvenile Nasopharyngeal Angiofibroma: Our Experience in a Tertiary Hospital
}

\author{
M. Mujtaba Khan ${ }^{1}$ L. Sudarshan $\operatorname{Reddy}^{2} \cdot$ N. Venkatram Reddy ${ }^{2}$. \\ D. Ranganath Swamy ${ }^{2}$
}

Received: 8 May 2017/Accepted: 6 September 2017/Published online: 22 November 2017

(C) Association of Otolaryngologists of India 2017

\begin{abstract}
Nasopharyngeal angiofibroma is a highly vascular tumor seen typically in young adolescent males. It is an uncommon tumor accounting for less than $0.5 \%$ of all head and neck tumors. This peculiar tumor is thought to arise from the area of the sphenopalatine foramen and it classically presents with progressive nasal obstruction and epistaxis. This is a retrospective study of angiofibroma treated at our hospital over 3 years. The study discusses about clinical features, management options and the role of embolization in reducing the intra-operative blood loss.
\end{abstract}

Keywords Angiofibroma · Nasopharynx · Vascular · Embolization

\section{Introduction}

Juvenile Nasopharyngeal Angiofibroma is a rare tumor occurring almost exclusively in young males. Though the tumor is considered benign, it has a propensity for local invasion and destruction. It is classically described as

L. Sudarshan Reddy

drlsudarshanreddy9@gmail.com

M. Mujtaba Khan

mujtaba354@gmail.com

N. Venkatram Reddy

venkatramreddyn@yahoo.com

D. Ranganath Swamy

rs.ranganathswamy@gmail.com

1 Department of ENT, Gandhi Medical College, Secunderabad, India

2 Department of ENT, Osmania Medical College, Hyderabad, India invading "through natural fissures and foramina". The pathogenesis of the angiofibroma has been a topic of much debate, but most authors agree that the tumor arises from the posterolateral wall of the nasal cavity and the adjoining supero-lateral nasopharyngeal wall and the sphenopalatine foramen is invariably involved. The tumor characteristically presents as progressive unilateral nasal obstruction and epistaxis. Diagnosis is made by clinical examination and imaging studies. Surgery is the gold standard treatment for angiofibroma [1]. Our study focuses on the presentation, surgical approach and more importantly the role of preoperative embolization in managing these highly vascular tumors.

\section{Materials and Methods}

The following study is a retrospective study done at ENT department in Government ENT Hospital, Hyderabad, India. From May 2010 to June 2013, 30 patients of Angiofibroma were operated, and are included in this study.

\section{Discussion}

All the 30 patients were males and no female case is reported in our series. The study includes patients between age of 9 and 24 years with a mean age of 16.2 years (Fig. 1). 15 patients fell in the most common age group of 16-20 years. Among all the symptoms epistaxis and nasal obstruction were the predominant symptoms in our study. Epistaxis was the most troublesome symptom for which the patients took medical advice. Amount of bleeding varied from 20 to $150 \mathrm{ml}$ in each episode, which was spontaneous. 


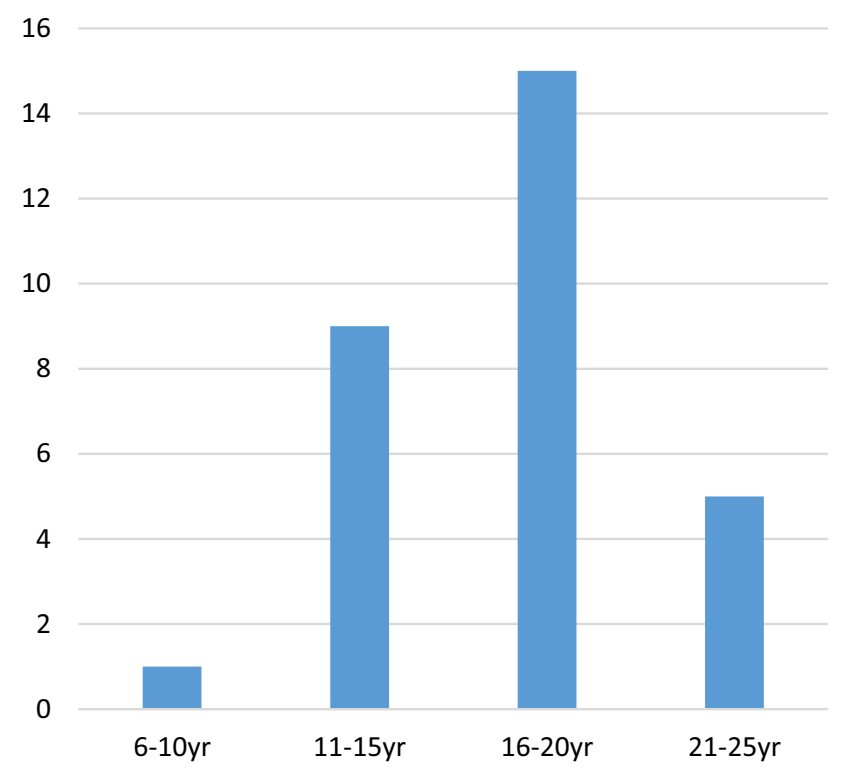

Fig. 1 Summary of the age group of nasopharyngeal angiofibroma. Numbers in bracket indicate number of patients

2 patients required post nasal packing in the emergency room to stop the bleeding. Nasal obstruction was progressive and much better tolerated symptom than epistaxis as majority of our patients presented with unilateral obstruction initially (Table 1). Nasal discharge was seen in 11 patients. 2 patients both recurrent cases, presented to us with isolated cheek swelling.

The tumor was evaluated by clinical examination, diagnostic nasal endoscopy and imaging. CT scan of nose and paranasal sinuses was performed in all the cases. The extension of the tumor was studied. In our study we applied the Fisch Staging system of the Angiofibroma [2] (Table 2).

The goal of tumor embolization is to occlude selectively the external carotid artery (ECA) feeders through intratumoral deposition of embolic material [3]. The embolic agents in common use are polyvinyl alcohol (PVA),

Table 1 Clinical presentation of angiofibroma

\begin{tabular}{lll}
\hline Symptoms/signs & No. of patients $(\mathrm{n}=30)$ & Percentage $(\%)$ \\
\hline Nasal obstruction & 22 & 73 \\
Epistaxis & 19 & 63 \\
Nasal discharge & 11 & 36 \\
Change in voice & 08 & 26 \\
Headache & 06 & 20 \\
Snoring & 05 & 16 \\
Face deformity & 03 & 10 \\
Cheek swelling & 02 & 6 \\
Intra-oral swelling & 02 & 6 \\
\hline
\end{tabular}

Embospheres, liquid embolic agents (glue, ethylvinyl alcohol copolymer [EVOH], or Onyx [ev3, Irvine, Calif]), gelatin sponge (Gelfoam; Phadia), and coils. The embolization is ideally performed $24-72 \mathrm{~h}$ before surgical resection to allow maximal thrombosis of the occluded vessels and prevent recanalization of the occluded arteries or formation of collateral arterial channels (Fig. 2).

The most common complication of embolization is facial pain. Serious complications occur in less than two percent of patients. These are usually related to particle reflux, poor technique, or non-visualization of dangerous anastomoses resulting in blindness or irreversible neurologic deficits [4].

\section{Results}

Nasopharynx was the most common site of extension in our study, with 22 patients having a nasopharyngeal extent of the tumor. Fifteen patients had Fisch II tumor at presentation. Two tumors had to be upstaged intraoperatively. (case nos. 6 and 26).

Among the 30 cases, pre-operative embolization with gelatin sponge (Gelfoam) was performed in 11 cases in our study. Embolization was performed by interventional radiologist $48 \mathrm{~h}$ prior to surgery in all the eleven patients. All the patients tolerated the procedure well, with 2 patients having facial pain for 2 days. Internal Maxillary artery was the main feeding vessel in 9 patients and in 2 patients both internal maxillary and ascending pharyngeal artery.

Based on the clinical and imaging findings, surgery was planned for the all patients. Different approaches were used for the surgery depending on the extent of the tumor. Four main approaches used in this study are Transpalatal, Endoscopic, Lateral Rhinotomy and combined approach (Transpalatal and Endoscopic) [5]. Weber Ferguson approach was done in 2 patients.

Among the eleven embolized patients, the intraoperative blood loss in 4 patients was insignificant and in the rest of patients ranged from 250 to $700 \mathrm{ml}$ with an average of $450 \mathrm{ml}$ [3-5]. Embolization reduced intra-operative blood loss from an average of $950 \mathrm{ml}$ in the non-embolized patients to $380 \mathrm{ml}$ in the embolized patients. Lateral rhinotomy approach was performed in 6 patients, and this approach gives excellent exposure in infratemporal fossa involvement (Fig. 3). Weber Ferguson approach was done in 2 patients with Stage IIIa and IIIb.

Endoscopic trans-nasal approach was performed in eleven patients [6,7]. This approach gives excellent exposure in Stage I and Stage II tumor (Fig. 4).

All thirty patients were followed up for a period of 2 years [Table 3]. Diagnostic nasal endoscopy was 
Table 2 Fisch staging of Juvenile nasopharyngeal angiofibroma

\begin{tabular}{ll}
\hline Stage I & Tumor limited to nasopharyngeal cavity, bone destruction negligible or limited to sphenopalatine foramen \\
Stage II & Tumor invading the pterygopalatine fossa or the maxillary, ethmoid or sphenoid sinus with bone destruction \\
Stage III & Tumor invading the infratemporal fossa or orbital region \\
& 1. Without intracranial involvement \\
& 2. With intracranial extradural involvement (parasellar) \\
Intracranial intradural involvement & 1. Without infiltration of the cavernous sinus, pituitary fossa or optic chiasm \\
& 2. With infiltration of the cavernous sinus, pituitary fossa or optic chiasm
\end{tabular}

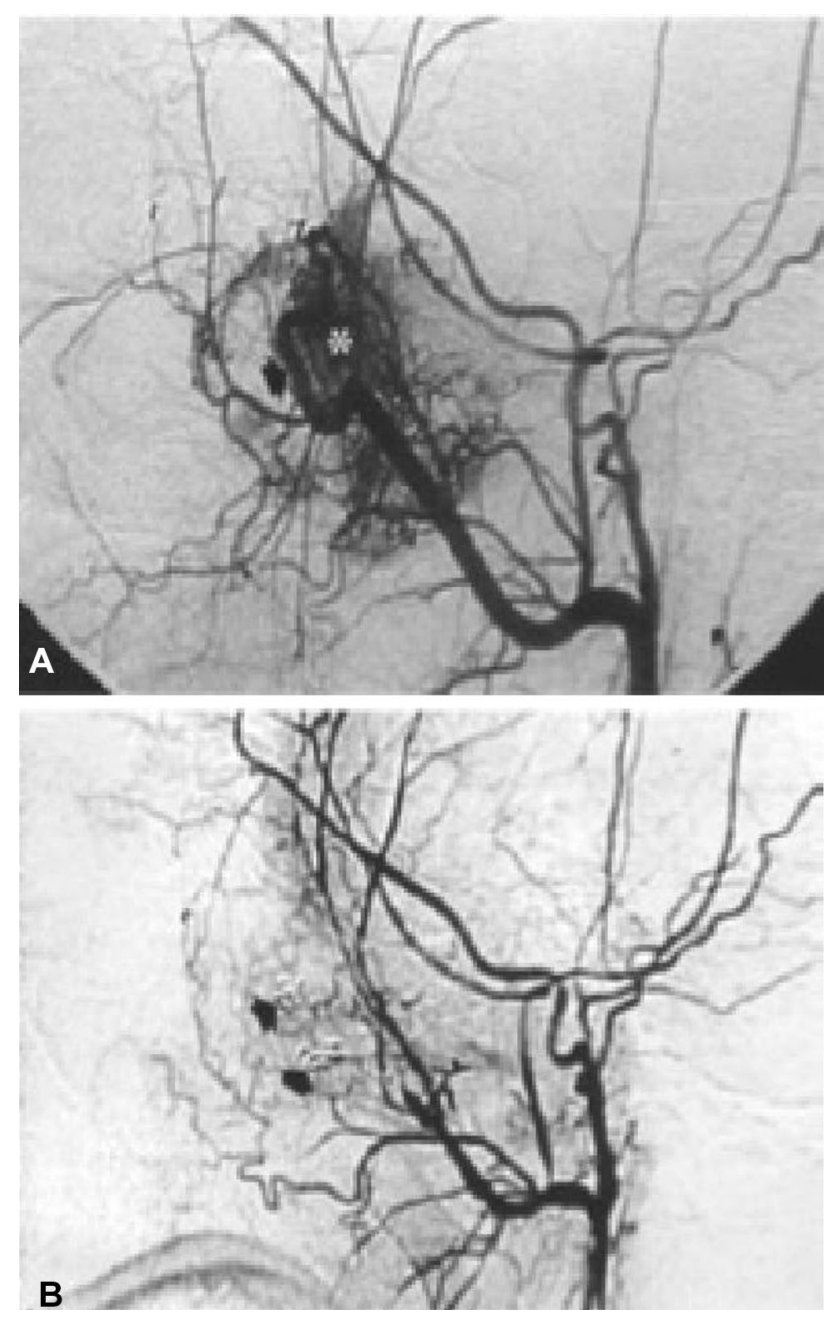

Fig. 2 a Before embolization (tumor blush can be seen). b After embolization

performed each month and a CT scan after 1 year. Recurrence was seen in three patients (case no. 3, 17 and 29 ) in the series. One patient who had been embolized and the other two, embolization was not performed. These patients presented with epistaxis after a mean duration of 4 months. All the three patients were later operated by Endoscopic approach.

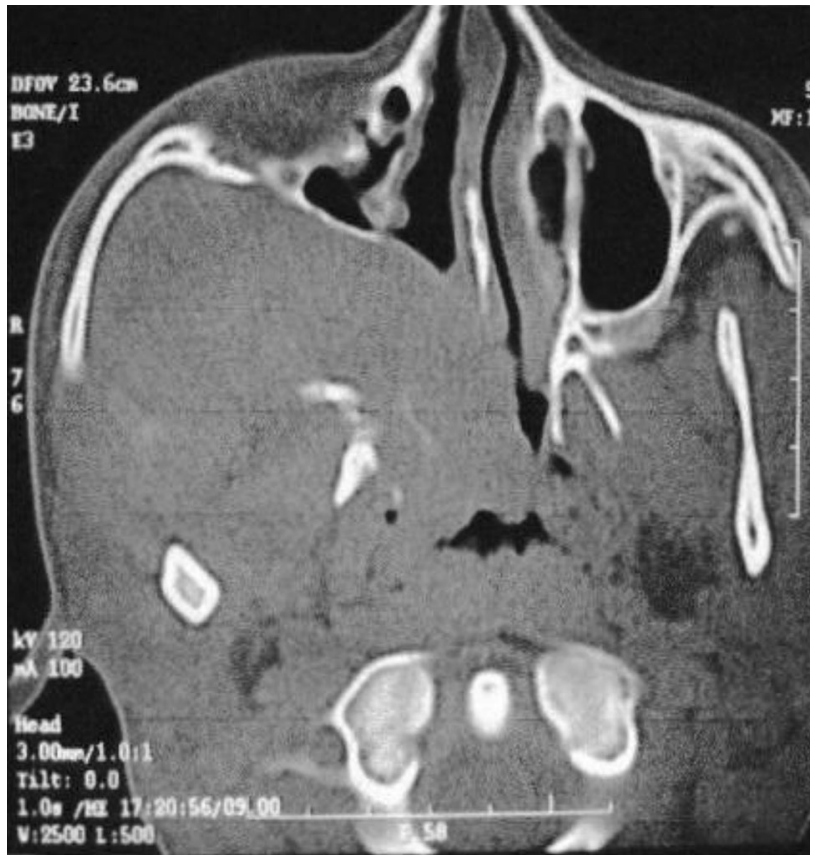

Fig. 3 CT Scan showing extension of angiofibroma into the infratemporal fossa

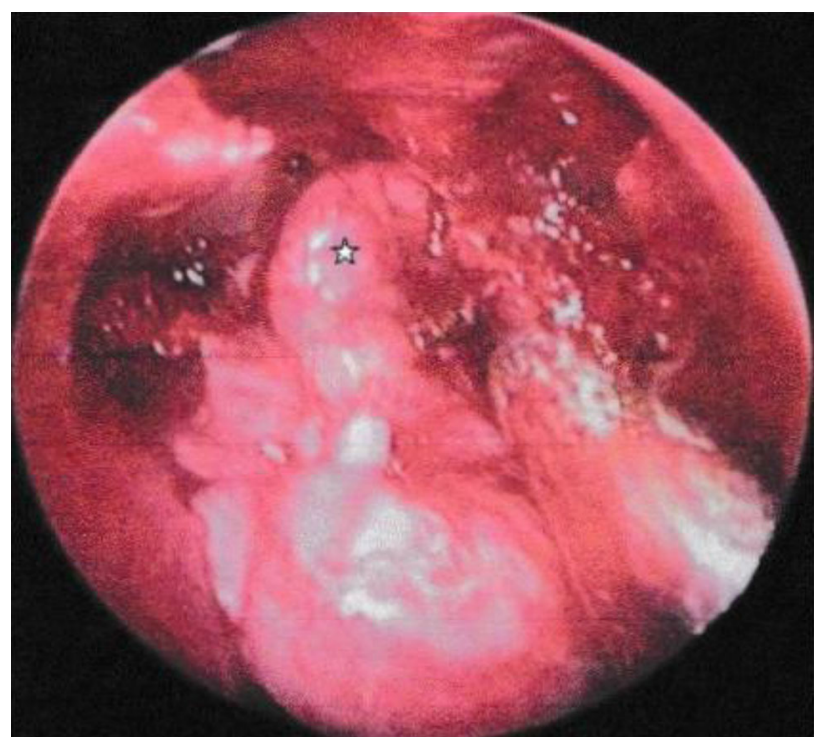

Fig. 4 Endoscopic transnasal approach in a Stage II tumor (embolized), intra-operative blood loss was insignificant 
Table 3 Summary of age group, pre-op stage, embolization and surgical approach in our study

\begin{tabular}{|c|c|c|c|c|}
\hline Case no. & Age & Pre-op stage & Embolization & Surgical approach \\
\hline 1 & 24 & IIIa & No & Lateral rhinotomy \\
\hline 2 & 17 & II & No & Transpalatal \\
\hline 3 & 16 & II & No & Endoscopic transnasal \\
\hline 4 & 21 & IIIb & No & Lateral rhinotomy \\
\hline 5 & 15 & II & No & Endoscopic transnasal \\
\hline 6 & 13 & $\mathrm{II}^{\mathrm{a}}$ & No & Lateral rhinotomy \\
\hline 7 & 16 & II & No & Endoscopic/transpalatal \\
\hline 8 & 18 & II & Yes & Endoscopic transnasal \\
\hline 9 & 20 & IIIa & Yes & Endoscopic/transpalatal \\
\hline 10 & 13 & II & Yes & Endoscopic \\
\hline 11 & 20 & IIIa & Yes & Lateral rhinotomy \\
\hline 12 & 17 & I & No & Transpalatal \\
\hline 13 & 1 & I & No & Transpalatal \\
\hline 14 & 20 & II & Yes & Endoscopic transnasal \\
\hline 15 & 14 & IIIb & Yes & Weber-Ferguson \\
\hline 16 & 20 & II & No & Endoscopic transnasal \\
\hline 17 & 16 & II & No & Transpalatal \\
\hline 18 & 15 & IIIa & Yes & Endoscopic/transpalatal \\
\hline 19 & 13 & II & No & Endoscopic transnasal \\
\hline 20 & 15 & IIIa & No & Lateral rhinotomy \\
\hline 21 & 17 & I & No & Transpalatal \\
\hline 22 & 17 & II & Yes & Endoscopic transnasal \\
\hline 23 & 19 & II & No & Endoscopic transnasal \\
\hline 24 & 22 & I & No & Endoscopic Transnasal \\
\hline 25 & 18 & IIIa & Yes & Lateral rhinotomy \\
\hline 26 & 23 & $\mathrm{II}^{\mathrm{a}}$ & No & Transpalatal \\
\hline 27 & 15 & II & No & Endoscopic transnasal \\
\hline 28 & 15 & IIIa & No & Weber-Ferguson \\
\hline 29 & 9 & II & Yes & Endoscopic-lateral rhinotomy \\
\hline 30 & 23 & II & Yes & Transpalatal \\
\hline
\end{tabular}

${ }^{a}$ Upstaged intraoperatively

\section{Conclusion}

Embolization pre-operatively reduces the blood loss, need for blood transfusions and decreases morbidity. Endoscopic approach obviates the need for an incision and gives excellent exposure in selected cases.

\section{Compliance with Ethical Standards}

Conflict of interest The authors declare that they have no conflict of interest.

Ethical Approval All procedures performed in studies involving human participants were in accordance with the ethical standards of the institutional and with the 1964 Helsinki declaration and its later amendments or comparable ethical standards.
Human and Animal Rights Animals were not involved in this study.

Informed Consent Informed consent was obtained from all individual participants included in the study.

\section{References}

1. Panda NK, Gupta G, Sharma S, Gupta A (2012) Nasopharyngeal angiofibroma-changing trends in the management. Indian $\mathrm{J}$ Otolaryngol Head Neck Surg 64:233-239

2. Fisch U (1983) The infratemporal approach for nasopharyngeal tumours. Laryngoscope 93(1):36-44

3. Lv MM, Fan XD, Su LX, Chen D (2013) Preoperative direct puncture embolization of advanced juvenile nasopharyngeal angiofibroma in combination with transarterial embolization: an 
analysis of 22 consecutive patients. Carrdiovasc Intervent Radiol 36:111-117

4. Trivedi M, Desai RJ, Potdar NA, Shinde CA, Ukirde V, Bhuta M, Nair AG (2015) Vision loss due to central retinal artery occlusion following embolization in a case of a Giant Juvenile nasopharyngeal angiofibroma. J Craniofac Surg 26(5):e451-e453. doi: 10.1097/SCS.0000000000001936

5. Boghani Z, Husain Q, Kanumuri V, Khan MN, Sangvhi S, Liu JK (2013) Juvenile nasopharyngeal angiofibroma: a systematic review and comparision of endoscopic, endoscopic-assisted, and open resection in 1047 cases. Laryngoscope 123:859-869

6. Andrade NA, Pinto JA, Nebrega MO (2007) Exclusively endoscopic surgery for Juvenile nasopharyngeal angiofibroma. Arch Otolaryngol Head Neck Surg 137:492-496

7. Petruson K, Rodriguez-Catarino M, Petruson B, Finizia C (2002) Juvenile nasopharyngeal angiofibromas: long term results in preoperative embolized and non-embolized patients. Acta Otollaryngol 122:96-100 